\title{
High-Power Supercontinuum Generation with Picosecond Pulses
}

\author{
Peter Horak, Kang Kang Chen, Shaif-ul Alam, Sonali Dasgupta, and David J. Richardson \\ Optoelectronics Research Centre, University of Southampton, Southampton SO17 1BJ, United Kingdom \\ e-mail:peh@orc.soton.ac.uk
}

\begin{abstract}
We investigate both theoretically and experimentally the properties of supercontinuum generation in the nearinfrared and visible regimes using high-power picosecond pulses propagating in a photonic crystal optical fiber. We discuss the effect of the pump wavelength relative to the fiber zero-dispersion wavelength on the conversion efficiency of pump power to shorter wavelengths. Finally, we consider picosecond supercontinuum generation in the presence of a mode cutoff when the pump is launched into a higher order fiber mode.
\end{abstract}

Keywords: Supercontinuum generation, high power fiber lasers, photonic crystal fibers.

\section{INTRODUCTION}

Spectral broadening of laser pulses, or supercontinuum generation (SCG), sometimes over several octaves has attracted significant attention over the last decade. In particular the invention and optimization of photonic crystal fibers (PCFs) have allowed researchers to control the dispersion properties of the guiding medium and to enhance nonlinear optical effects for ever increasing supercontinuum bandwidths and powers [1]. The introduction of highly nonlinear soft glass PCFs has further aided this development [2].

Much of the work on SCG has been focused on pumping with femtosecond lasers which allow for high peak powers and thus spectral broadening over short lengths of optical fiber (typically of the order of $1 \mathrm{~m}$ ). SCG in this regime is mainly based on soliton effects, such as soliton fission, soliton Raman self frequency shifting, and emission of dispersive waves by solitons. In order to achieve very high average power levels, on the other hand, longer laser pulses or even continuous wave pumping are required. For example, $28 \mathrm{~W}$ and $50 \mathrm{~W}$ of $\mathrm{CW}$ supercontinuum have been reported [3]. In this operating regime, the basic mechanism of SCG relies on modulation instability, rather than soliton effects.

Here we investigate SCG using a high power 20ps pulsed source at $1060 \mathrm{~nm}$ wavelength giving up to $39 \mathrm{~W}$ of supercontinuum output [4]. We compare these experimental results with numerical simulations of picosecond pulse propagation, in particular focusing on the power level of light shifted to the short wavelength side of the pump. We finally investigate the effects of a mode cutoff at long wavelengths, as realized in SCG in higher order fiber modes. Such a system has recently been proposed as a means to enhance short wavelength conversion in the fs pumping regime [5]. Our simulations suggest fundamentally different behavior in the ps regime.

\section{EXPERIMENTAL RESULTS}
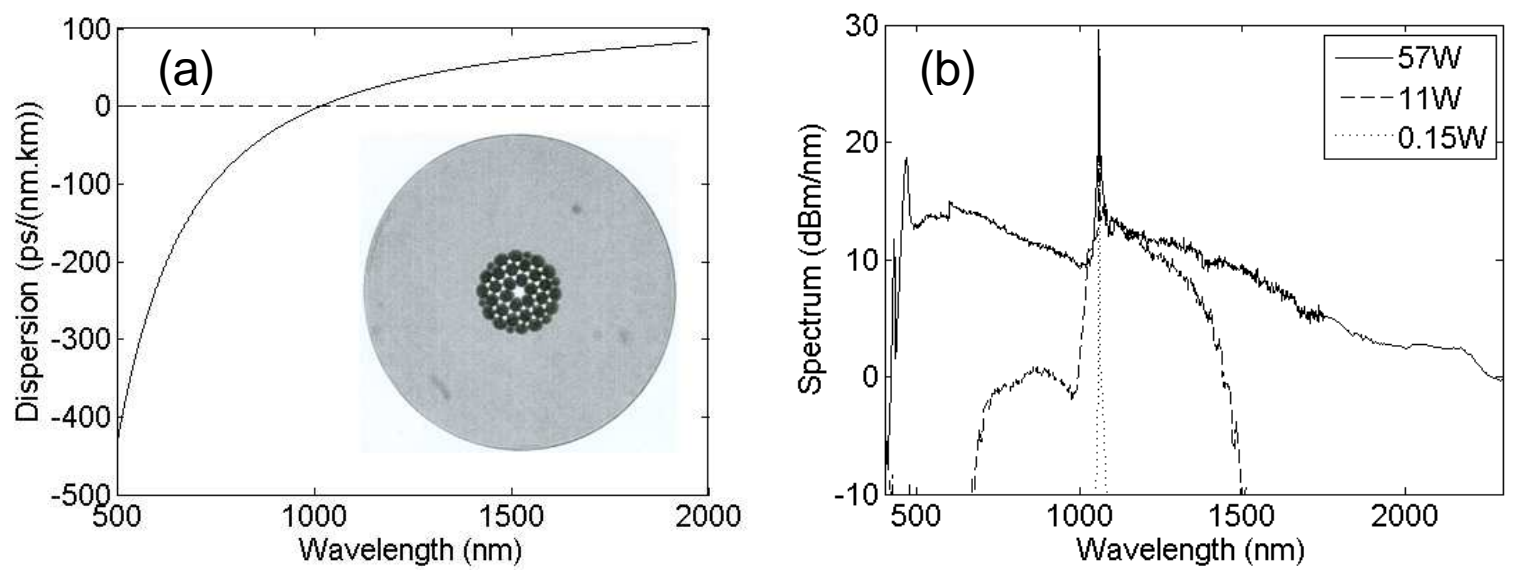

Figure 1. (a) Dispersion profile and structure of the fiber used for the experiments and the simulations. (b) Measured supercontinuum spectra at different input power levels. The spectrum at 57W was measured with an optical spectrum analyzer up to 1750nm wavelength and with a monochromator and a PbS detector at longer wavelengths.

In our experiments [4] the laser source is an Yb-doped fiber MOPA with a 21ps gain-switched seed delivering an average power of up to $100 \mathrm{~W}$ at a repetition rate of $114.8 \mathrm{MHz}$ and at a wavelength of $1060 \mathrm{~nm}$. The corresponding maximum pulse energy and peak power are $0.8 \mu \mathrm{J}$ and $39 \mathrm{~kW}$, respectively. The pump is launched into a $2 \mathrm{~m}$ long PCF fabricated by the Central Glass and Ceramic Research Institute Kolkata, India; the fiber structure is shown in Fig. 1(a). The fiber has a pitch $(\Lambda)$ of $5.29 \mu \mathrm{m}$ and a ratio of air hole diameter to pitch 
$\mathrm{d} / \Lambda=0.956$. The dispersion profile of the fiber, Fig. 1(a), exhibits a zero-dispersion wavelength (ZDW) at $1012 \mathrm{~nm}$, such that the pump wavelength lies in the anomalous dispersion regime.

In Fig. 1(b) we show the measured supercontinuum spectra generated with this system for different pump power levels. The output spectra exhibit a transmitted peak at the pump wavelength, but otherwise show a very flat spectrum covering the range from $400 \mathrm{~nm}$ to $2300 \mathrm{~nm}$ at maximum pump power. Flatness in the visible region is better than $10 \mathrm{~dB}$ and the power density is $\sim 31.7 \mathrm{~mW} / \mathrm{nm}$ in the visible, which is believed to be a record level. The total average output power is $39 \mathrm{~W}$ for an input power of $57 \mathrm{~W}$. We note that the spectral shape is similar to the one observed with a $20 \mathrm{~m}$ PCF and a ns microchip laser at much lower powers [6].

\section{NUMERICAL SIMULATION OF PICOSECOND PULSE PROPAGATION}

In the following we present results of numerical simulations of high power picosecond pulse propagation in the fiber described in the previous section. To this end we solve the generalized nonlinear Schrödinger equation [1], including the full dispersion profile of the fiber as well as Raman and Kerr nonlinearities and self-steepening terms, using an adaptive step-size split-step Fourier technique which we previously employed in the fs regime [7]. The input pulses are assumed to have a Gaussian profile with a full width at half maximum of 20ps. Because of the narrow linewidth of such pulses, a fine spectral grid is required for the simulations, and thus a relatively large number $\mathrm{N}=65536$ of discrete wavelengths is used to cover the full spectral range covered by the supercontinuum output. In order to reduce the simulation times, we restrict the fiber length to $1 \mathrm{~m}$ and pump peak powers to $20 \mathrm{~kW}$.
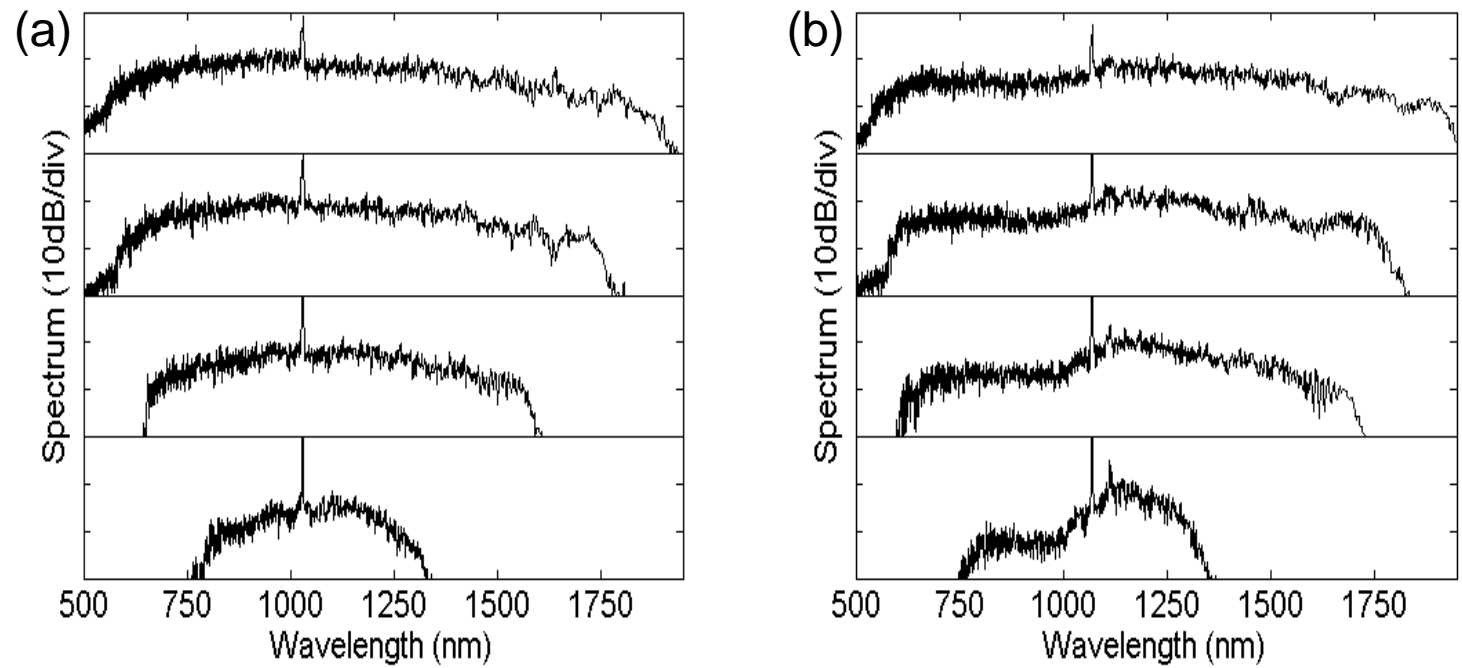

Figure 2. Simulated supercontinuum generation over $1 \mathrm{~m}$ fiber length with 20ps pulses of $5 \mathrm{~kW}, 10 \mathrm{~kW}, 15 \mathrm{~kW}$, and $20 \mathrm{~kW}$ peak power (from bottom to top) at a pump wavelength of (a) $1030 \mathrm{~nm}$ and (b) $1070 \mathrm{~nm}$.

Two sets of simulations at different pump peak power levels from $5 \mathrm{~kW}$ to $20 \mathrm{~kW}$ are shown in Fig. 2. The two sets differ only in the choice of pump wavelength: 1030nm for Fig. 2(a) and 1070nm for Fig. 2(b). As expected, we find larger broadening at higher input powers. In all cases the resulting spectra are extremely flat, with power densities varying by less than $10 \mathrm{~dB}$ over the supercontinuum wavelength range. The spectra at 1070nm pumping closely resemble the experimental results of Fig. 1, in particular at lower powers. At high powers, the experimental spectrum shows a slope towards longer wavelengths which is absent in the simulations. The reason for this discrepancy is yet unknown.

Comparing the results for the two pump wavelengths, it is evident that pumping closer to the ZDW (at $1012 \mathrm{~nm}$ ) leads to even improved spectral flatness. Simultaneously, a larger fraction of power is transferred from the pump to shorter wavelengths in this case. Vice versa, pumping deeper in the anomalous dispersion regime, Fig. 2(b), leads to enhanced power transfer to the longer wavelength components of the supercontinuum spectrum. We attribute this to more prominent soliton effects in this pumping regime after the initial phase of modulation instability has broken up the ps pump pulse. In order to demonstrate this dependence on the pump wavelength more clearly, we plot the ratio of the integrated output power in the wavelength band between $500 \mathrm{~nm}$ and the pump wavelength relative to the input pump power in Fig. 3. We observe a marked reduction of power conversion efficiency towards short wavelengths as the pump wavelength is tuned away from the ZDW. Moreover, higher pulse peak powers, and thus larger nonlinear effects, also lead to better conversion efficiencies. At peak powers of $20 \mathrm{~kW}$ short wavelength conversion efficiencies up to $43 \%$ are observed for pumping near the ZDW compared to only $26 \%$ for pumping at $\sim 60 \mathrm{~nm}$ inside the anomalous dispersion regime. As a final remark we note that when pumping below the zero-dispersion wavelength, i.e., in the normal dispersion regime, our 
simulations show that the SCG process is initially dominated by the generation of widely separated spectral sidebands through four-wave mixing, which significantly deteriorates the flatness of the output spectrum.

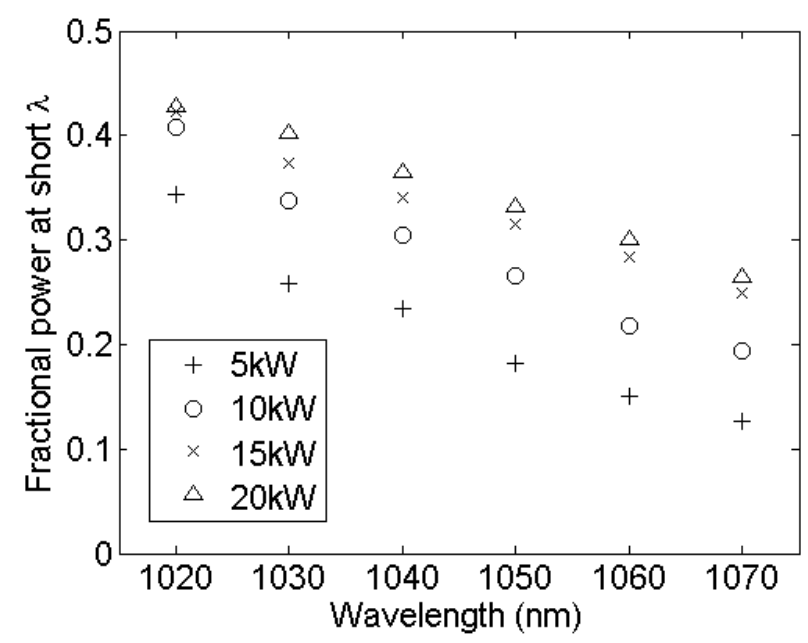

Figure 3. Ratio of the supercontinuum output power confined in the wavelength range between 500nm and the pump wavelength relative to the input pump power for different pump wavelengths and peak powers.

\section{SUPERCONTINUUM GENERATION IN MODES WITH LONG-WAVELENGTH CUTOFF}

Recent work has suggested that supercontinuum generation with fs pulses exhibits improved short wavelength conversion efficiencies when a multimode fiber is pumped in a higher order mode, where the mode cutoff prevents the transfer of power to long wavelengths [5]. Here we consider the same idea for ps pumping. We thus present results of numerical simulations where for simplicity we keep the same dispersion profile shown in Fig. 1(a) for the fundamental fiber mode, but mimic an effective mode cutoff by introducing large propagation losses of $100 \mathrm{~dB} / \mathrm{m}$ for wavelengths $>1270 \mathrm{~nm}$. Coupling of light to other modes of a multimode fiber [8] is ignored in these simulations.

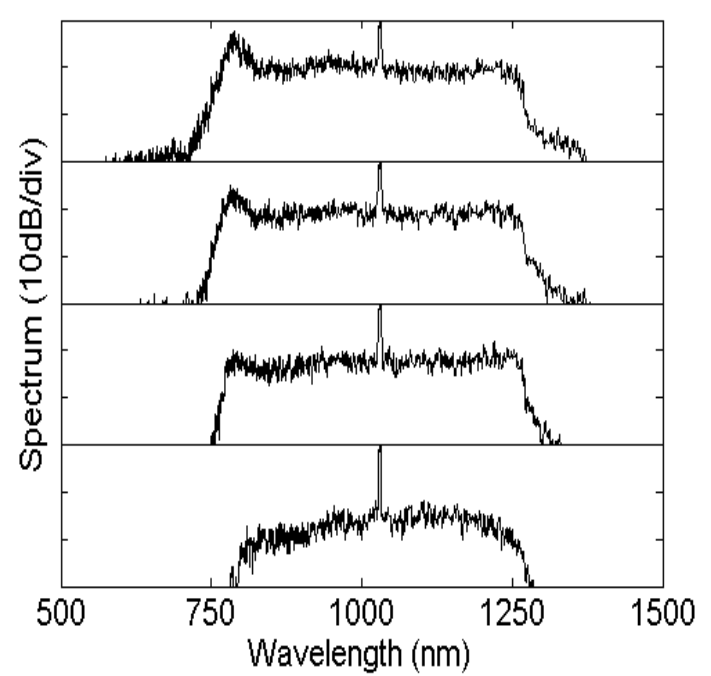

Figure 4. Simulated supercontinuum generation over $1 \mathrm{~m}$ fiber length with $20 \mathrm{ps}$ pulses of $5 \mathrm{~kW}, 10 \mathrm{~kW}, 15 \mathrm{~kW}$, and $20 \mathrm{~kW}$ peak power (from bottom to top) at a pump wavelength of $1030 \mathrm{~nm}$ with a mode cutoff at 1270nm.

Figure 4 shows the results of these simulations for various power levels with a pump wavelength of $1030 \mathrm{~nm}$, the same as in Fig. 1(a). The simulated supercontinuum spectra clearly show the wavelength cutoff, beyond which light cannot propagate in the mode. However, there is also a similarly steep edge on the short wavelength side of the pump approximately symmetric in position to the mode cutoff. The generated spectrum thus assumes a flat top shape that is nearly independent of the pump power, with extinction ratios of the order of $20 \mathrm{~dB}$. The efficiency of power transfer to short wavelengths is found to increase from $22 \%$ at $5 \mathrm{~kW}$ peak power to $33 \%$ at $20 \mathrm{~kW}$ and is thus smaller than the corresponding values without mode cutoff shown in Fig. 3. This significant difference in behavior compared to the fs regime [5] is explained by the nature of the SCG process for ps pulses. Modulation instability is based on a degenerate four-wave mixing process where two pump photons are 
converted into one photon each in a short-wavelength and in a long-wavelength sideband. Beyond the mode cutoff, the long-wavelength photon is quickly lost from the fiber, which prevents amplification and gain of the entire FWM process including the otherwise exponential growth of the short wavelength sideband.

\section{CONCLUSIONS}

We have presented experimental results of supercontinuum generation with picosecond pulses in a photonic crystal fiber, resulting in a $39 \mathrm{~W}$ supercontinuum output at record spectral densities in the visible regime. Numerical simulations show a dependence of the short wavelength conversion efficiency on the position of the pump wavelength with respect to the zero-dispersion wavelength of the fiber. Short wavelength conversion efficiencies close to $50 \%$ are predicted for pumping at high power near the ZDW. We finally investigated the effect of a mode cutoff on ps supercontinuum generation. This results in flat-top supercontinuum spectra symmetric around the pump wavelength, but no improved conversion efficiencies could be achieved.

\section{REFERENCES}

[1] J.M. Dudley, G. Genty, S. Coen: Supercontinuum generation in photonic crystal fiber, Rev. Mod. Phys., vol. 78, pp. 1135-1184, Oct.-Dec. 2006.

[2] J.H.V. Price, et al.: Mid-IR supercontinuum generation from nonsilica microstructured optical fibers, IEEE J. Sel. Top. Quantum Electron., vol. 13, pp. 738-749, May/June 2007.

[3] J.C. Travers, et al.: Visible supercontinuum generation in photonic crystal fibers with a $400 \mathrm{~W}$ continuous wave fiber laser, Opt. Express, vol. 16, pp. 14435-14447, Sept. 2008.

[4] K.K. Chen, et al:: Picosecond fiber MOPA pumped supercontinuum source with $39 \mathrm{~W}$ output power, Opt. Express, vol. 18, pp. 5426-5432, Mar. 2010.

[5] R. Cherif, et al:: Supercontinuum generation by higher-order mode excitation in a photonic crystal fiber, Opt. Express, vol. 16, pp. 2147-2152, Feb. 2008.

[6] J.M. Stone, J.C. Knight: Visibly "white" light generation in uniform photonic crystal fiber using a microchip laser, Opt. Express, vol. 16, pp. 2670-2675, Feb. 2008.

[7] F. Poletti, P. Horak: Dynamics of femtosecond supercontinuum generation in multimode fibers, Opt. Express, vol. 17, pp. 6134-6147, Apr. 2009.

[8] F. Poletti, P. Horak: Description of ultrashort pulse propagation in multimode optical fibers, J. Opt. Soc. Am. B, vol. 25, pp. 1645-1654, Oct. 2008. 